\title{
Coronary occlusive disease and late graft failure after cardiac transplantation
}

P A Mullins, N R Cary, L Sharples, J Scott, D Aravot, S R Large, J Wallwork, P M Schofield

\begin{abstract}
Objective-Coronary occlusive disease is the main cause of late mortality after cardiac transplantation. It has both similarities and differences compared with conventional atherosclerotic coronary disease. The pathophysiology of late graft failure from coronary occlusive disease is unclear at present. We reviewed the experience of this disorder in our cardiac transplant programme.

Design-A retrospective analysis of angiographic and pathological data.

Setting-A regional cardiothoracic centre and transplant unit.

Patients-Of a population of 383 orthotopic cardiac transplant recipients operated upon between January 1979 and June 1990, 447 coronary angiograms were available for review in 193 patients. Thirteen of a possible 18 results of post mortem examinations from patients dying from coronary occlusive disease were available.
\end{abstract}

Main outcome measure-Coronary occlusive disease was defined as any evidence of disease on coronary angiography. Post mortem examinations were performed with standard techniques.

Results The angiographic prevalence of coronary occlusive disease was $3 \%$ (1/32 patients) and 40\% (19/47 patients)

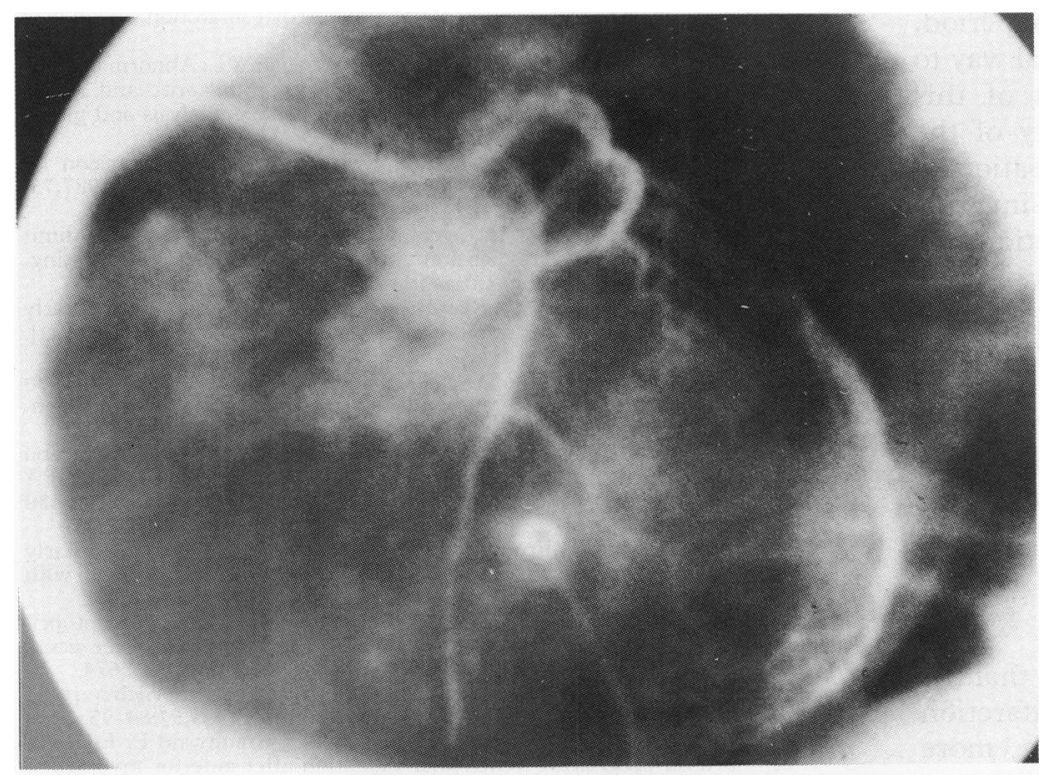

Figure 1 Left anterior oblique view showing diffuse irregularity and terminal occlusion of left anterior descending and left circumflex coronary artery. at one and five years respectively. Twenty six grafts failed due to coronary occlusive disease compared with 132 graft failures from all causes during this period. Acute thrombosis was present in a large vessel in seven of 13 fatal cases undergoing necropsy (54\%). Noticeable large vessel involvement with disease in smaller distal vessels was present in four patients $(31 \%)$. The remaining two patients $(15 \%)$ had small vessel disease alone. Twelve of the 13 patients had significant cardiomegaly (cardiac weight $\geqslant 400 \mathrm{~g}$ ) with a mean weight of 510 (range 370-740) g.

Conclusion-Coronary occlusive disease is the main late complication after cardiac transplantation. A combination of coronary thrombosis, ischaemia from stenoses of large and small coronary vessels, and cardiomegaly contribute to the graft failure of these patients.

(Br Heart J 1992;68:260-5)

Coronary occlusive disease is the main cause of death in orthotopic cardiac transplant recipients more than one year after cardiac transplantation. ${ }^{1}$ This disease differs from conventional atherosclerosis in that it is tubular, concentric, diffuse, and often affects smaller coronary vessels. ${ }^{23}$ It may not only develop proximally in larger coronary arteries as in conventional atherosclerosis, but may be distal and obliterative (fig 1). Often it appears as a combination of these types of lesion and characteristically progresses rapidly. ${ }^{4}$ As patients are denervated at the time of operation, cardiac ischaemia can develop without symptoms until severe cardiac dysfunction and death occurs. ${ }^{5}$

Serial coronary angiography has been the main method of assessing coronary occlusive disease after operation. It is performed to detect coronary disease and left ventricular dysfunction, and to plan appropriate treatment. Also it is used as a research tool for studying a form of accelerated coronary disease in humans. What remains unclear is the prognostic importance of the various coronary angiographic lesions found in patients with coronary occlusive disease, and what is the mechanism of graft failure and death.

We documented the incidence and time to occurrence of any form of coronary occlusive disease as apparent from coronary angiography at our hospital. We reviewed the available data from post mortem examination 
of patients who died during the study, and examined some of the processes which led to graft failure.

\section{Patients and methods}

A total of 383 patients received their first cardiac transplant at Papworth Hospital between January 1979 and June 1990. Most $(88 \%)$ of the recipients were male $(n=338)$. The mean age of these patients was 44 (range 6-63) years at the time of operation. Ischaemic heart disease $(53 \%)$ and dilated cardiomyopathy $(43 \%)$ were the main indications for transplantation.

\section{IMMUNOSUPPRESIVE TREATMENT}

The first 29 patients in the cardiac transplant programme received azathioprine and prednisolone only. Cyclosporin was introduced at Papworth Hospital in March 1982 and was used in 121 patients in conjunction with either azathioprine or prednisolone for the next four years (double treatment). Triple treatment, combining cyclosporin with azathioprine and prednisolone, was started in April 1986. Oral steroids are gradually withdrawn, if possible, in the triple treatment group starting three months after operation.

\section{ANGIOGRAPHY PROTOCOL}

Up to March 1983, patients underwent annual coronary angiography. After this time, a different protocol was devised. The first coronary angiogram is now performed two years after operation. If coronary occlusive disease is detected, annual angiography is performed and if not, the next angiogram is performed at four years. After four years, all patients undergo annual angiography.

Angiograms were assessed serially for each patient by two observers blinded to the clinical history. Coronary occlusive disease was defined as any sign of disease on angiography. Coronary disease was graded according to the diameter of the stenosis of the most severe lesion compared with an adjacent healthy artery. The primary coronary arteries were defined as the left anterior descending coronary artery, left circumflex coronary artery, and right

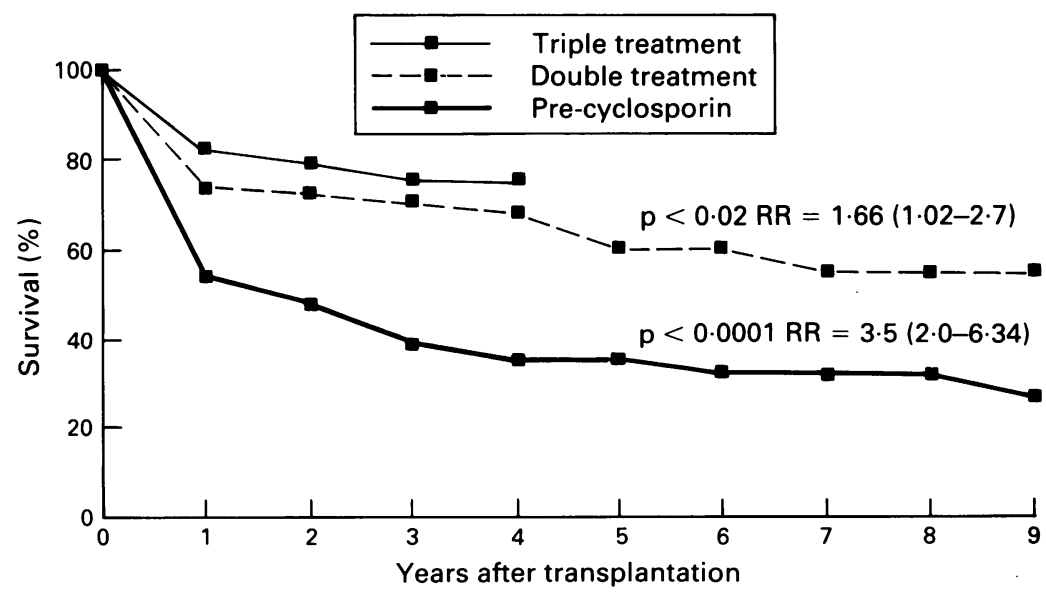

Figure 2 Acturial survival after orthotopic cardiac transplantation according to type of immunosuppressive treatment. $R R$, relative risk; numbers in parentheses are ranges. coronary artery. Their main branches were classified as secondary coronary arteries (first and second diagonal, first and second obtuse marginal, and posterolateral or posterior descending branch of the right coronary artery). Initially, attempts to assess disease angiographically in smaller (tertiary) branches of these arteries were made. Without annual serial films, the consistent ability to detect disease affecting these vessels in all patients was not possible. ${ }^{4}$ Therefore this approach was abandoned. Significant coronary disease was defined as a lesion producing a greater than $50 \%$ reduction in intraluminal arterial diameter.

\section{PATHOLOGY}

Efforts to procure the heart for pathological examination were made in all patients who died. After fixation in buffered formalin solution, the coronary arteries were examined throughout their lengths. Serial transverse sections were taken at regular intervals and paraffin sections were stained with haematoxylin and eosin. Both large (primary and secondary) and small (tertiary) vessels were examined by light microscopy. Cardiomegaly was defined as a post mortem heart weight of $\geqslant 400 \mathrm{~g}^{6}$ The diagnosis of death due to coronary occlusive disease was made in several patients at post mortem examinations performed at other hospitals. Unless the heart was released for examination here, this data is not included in our study.

\section{STATISTICAL METHODS}

Statistical analysis was performed with the $B M D P$ statistics package. Results are expressed as mean (ranges) for continuous measurements. Actuarial survival was calculated by the life table method. The risk of death associated with improved immunosuppressive treatment was assessed by Cox regression analysis and tested with the likelihood ratio test.

\section{Results}

The overall survival figures have improved with changes in immunosuppressive treatment (fig 2). Most patients undergoing coronary angiography received cyclosporin treatment. No documented change in the incidence of coronary occlusive disease has occurred, however, with the various developments in immunosuppressive treatment here or elsewhere as yet. ${ }^{4}$ This is shown by the similarity in slope of the decline in survival curves after the first year in each immunosuppressive treatment group (fig 2).

\section{CORONARY ANGIOGRAPHY}

We reviewed 447 coronary angiograms in 193 patients. Ninety nine patients had two or more coronary angiograms. Coronary occlusive disease was present in 3\% (1/32) of patients undergoing coronary angiography by the first year, and $40 \%(19 / 47)$ at five years after operation. Figure 3 shows freedom from coronary occlusive disease as assessed on coronary angiography over seven years from transplantation. Coronary collateral vessels were seen to 
Figure 3 Freedom from coronary occlusive disease as indicated by coronary angiography.

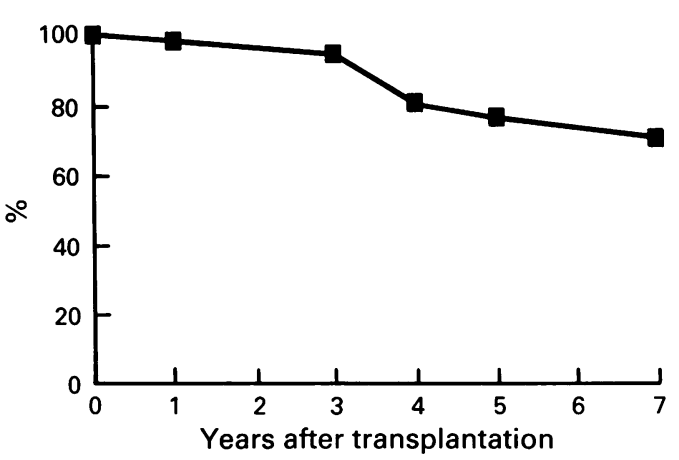

supply $12 \%(3 / 26)$ of the occluded coronary arteries in our study.

The severity of coronary stenosis produced by coronary occlusive disease was usually modest. For example, of the angiograms showing coronary occlusive disease in the left anterior descending coronary artery, 55\% (61/ $110)$ showed lesions that decrease the diameter of affected vessels by $<25 \%$ of the diameter of the adjacent arteries. Only $12 \%$ of the cine films (13/110) showed lesions of $>50 \%$ stenosis in the left anterior descending coronary artery. Therefore, the degree of luminal obstruction was usually not severe. The disease, however, often affects different portions of the same artery as well as other vessels. This pattern of distribution of the disease makes accurate anatomical description difficult.

\section{GRAFT FAILURE}

Coronary occlusive disease caused failure of 26 grafts $(20 \%)$ compared with 132 graft losses from all causes during this period. Most of these patients died later than one year after operation (table 1). Thirteen patients who had undergone coronary angiography died and the heart was available for full examination. Seven patients underwent eight cardiac retransplantation operations. The remaining five patients died and had necropsies, but the heart was not examined at this hospital.

The mean age of all patients at the time of graft failure was 39 (range 23-57) years. The median time from the identification of coronary occlusive disease angiographically to death in the group dying of coronary occlusive disease was $2 \cdot 3$ (range $0 \cdot 1-5 \cdot 6$ ) years. Twelve of the 13 patients who died and had post mortem examinations had considerable cardiomegaly (weight $\geqslant 400 \mathrm{~g}$ ) with a mean overall weight of 507 (range 370-740) g.

Coronary angiograms were performed before graft failure in 18 of the patients who had pathology data available (table 2). Coronary angiography tended to underestimate the severity of the stenosis in coronary occlusive disease found at post mortem examination. It is, however, difficult to assess accurately the severity of stenosis in post mortem samples that have not been pressure perfused. This structural comparison is therefore limited in value.

\section{MORTALITY}

Thirteen patients died and underwent detailed post mortem examination. The mode of death was acute thrombosis on a significant lesion affecting a primary or secondary vessel in seven cases (table 2). In two of these patients there was evidence of old thrombus in different vessels to the ones affected by the acute thrombus. There was post mortem evidence of previous non-transmural myocardial infarction in two cases, and a recent infarction in one patient who died.

In four of the 13 patients undergoing post mortem examination there was histological evidence of coronary disease in small tertiary branches, as well as the large primary and secondary coronary vessels. Three patients died with diffuse involvement of small tertiary vessels alone. When disease in small coronary vessels was the only cause of death, coronary angiography did not detect coronary disease. It was performed in two of these cases before death, and appeared normal, and was not done in the remaining patient as death occurred before the first routine coronary angiogram was performed.

The time from the first sign of coronary disease to death progressively decreased as more primary coronary arteries were diseased, but the differences were not statistically significant (fig 4). Longer follow up in more patients is required to clarify this. Coronary occlusive disease was not detected on coronary angiography in $107(55 \%)$ patients at any stage during during the study period. Eight of these patients have died: two of coronary occlusive disease, two of malignancy, two of sudden death, and two of other causes.

\section{RETRANSPLANTATION}

Seven patients were retransplanted for coronary occlusive disease. One patient required a further retransplant for recurrent coronary disease. The median time from transplantation to reoperation was 3.6 (range $0 \cdot 3-8 \cdot 8$ ) years. These patients had progressive coronary disease that allowed elective reassessment for reoperation. Only three of these patients had coronary angiography performed on their second graft during the study period. Therefore there is insufficient data to assess angiographic recurrence of coronary disease in retransplanted patients.
Table 1 June 1990

\begin{tabular}{|c|c|c|c|}
\hline \multirow[b]{2}{*}{ Causes of death } & \\
\hline & $0-90$ days & 90 days -1 year & $>1$ year \\
\hline Rejection & 23 & 9 & 1 \\
\hline Infection & 21 & 8 & 3 \\
\hline Coronary occlusive disease & 0 & 2 & 26 \\
\hline Sudden cardiac death & 1 & 1 & 3 \\
\hline Donor heart failure & 6 & 0 & 0 \\
\hline Stroke & 4 & 1 & 0 \\
\hline High pulmonary resistance & 4 & 0 & 0 \\
\hline Multifactorial & 4 & 0 & 0 \\
\hline Lymphoma & 1 & 0 & 2 \\
\hline Malignancy & 0 & 1 & 5 \\
\hline Abdominal complications & 2 & 0 & 0 \\
\hline Operative bleeding & 1 & 0 & 0 \\
\hline Unknown & 1 & 1 & 1 \\
\hline Total & 68 & 23 & 41 \\
\hline
\end{tabular}


Table 2 Post mortem data

\begin{tabular}{|c|c|c|c|c|c|c|}
\hline $\begin{array}{l}\text { Patient } \\
\text { No }\end{array}$ & $\begin{array}{l}\text { Age } \\
(y)\end{array}$ & $\begin{array}{l}T T F \\
(y)\end{array}$ & $A n$ & $P M$ & Event & Wt of heart $(g)$ \\
\hline 1 & 29 & 3 & NCA & 2VD T & death & 440 \\
\hline 2 & 49 & 3.5 & MINLVD & 1VDT & death & 400 \\
\hline 3 & 50 & $1 \cdot 7$ & NCA & 1VD & death & 600 \\
\hline 4 & 52 & $5 \cdot 9$ & 3VD & LMain T 3VD MI(old) & death & 510 \\
\hline 5 & 23 & 8 & MINLVD & 2VD T & death & 580 \\
\hline 6 & 30 & $2 \cdot 5$ & MINLVD & 1VD Told & death & 480 \\
\hline 7 & 46 & $0 \cdot 7$ & NCA & SVD & death & 480 \\
\hline 8 & 44 & 4 & $\mathrm{Cx}$ & 3VD & death & 500 \\
\hline 9 & 45 & 4 & LVD-OM & 1VD-T & death & 480 \\
\hline 10 & 52 & $4 \cdot 5$ & NAD & SVD & death & 740 \\
\hline 11 & 26 & $2 \cdot 8$ & $3 \mathrm{VD}$ & 3VD Told old and new MIs & death & 390 \\
\hline 12 & 36 & 1.9 & $3 \mathrm{VD}$ & 1VD-T MI & death & 610 \\
\hline 13 & 28 & 1 & NCA & 1VD & death & 670 \\
\hline 14 & 25 & 3 & NCA & SVD & RETX & 440 \\
\hline 15 & 35 & $8 \cdot 8$ & 2VD SVD & 3VD & RETX & 600 \\
\hline \multirow[t]{2}{*}{16} & 34 & $3 \cdot 1$ & $2 \mathrm{VD}$ & 3VD & RETX & 370 \\
\hline & & $1 \cdot 2$ & 2VD & $3 \mathrm{VD}$ & RETX & 370 \\
\hline 17 & 21 & $4 \cdot 4$ & NCA & 2VD & RETX & 480 \\
\hline 18 & 36 & $5 \cdot 2$ & 3VD & $3 \mathrm{VD}$ & RETX & 545 \\
\hline 19 & 26 & $4 \cdot 1$ & 3VD & 3VD & RETX & 510 \\
\hline 20 & 57 & 0.3 & NCA & SVD & RETX & 470 \\
\hline
\end{tabular}

sCA, no coronary arteriogram available; VD, vessel disease; T, fresh thrombus; MINLVD, minor disease in large epicardial coronary artery; SVD, OM, large vessel disease obtuse marginals; RETX, retransplanted; MI, myocardial infarction.

\section{Discussion}

Coronary occlusive disease probably occurs as a result of responses to immunologically mediated vascular injury. ${ }^{7}$ It may be related to the arteriopathy seen in other transplanted solid organ grafts. ${ }^{8}$ Higher incidences of myocardial cellular rejection ${ }^{910}$ and vascular (humoral) rejection ${ }^{11}$ have been reported in patients developing coronary occlusive disease. Hyperlipidaemia, often exacerbated by steroid ${ }^{12}$ and cyclosporin treatment, ${ }^{13}$ is probably an important cofactor in the development of coronary occlusive disease. High total cholesterol, high LDL cholesterol, ${ }^{13}$ and low HDL cholesterol concentrates, ${ }^{14}$ and hypertryglyceridaemia ${ }^{15}$ have been implicated in some studies. Cytomegalovirus infection has been suggested as a potential cause of the disorder. ${ }^{16}$ The relation between virus infection and coronary occlusive disease has not been found at other centres. ${ }^{14}$ There is no evidence, as yet, of cytomegalovirus causing direct coronary artery damage. Other factors have been reported as being associated with development of disease (table 3 ). The aetiology is unclear at present. No single factor appears to be so important that it stands out, because of the few patients studied. It is probable that coronary occlusive disease is a manifestation of chronic vascular rejection in combination with other cofactors. ${ }^{22}$ Until the pathogenesis

Figure 4 Survival from first angiographic evidence of coronary occlusive disease according to number of vessels affected.

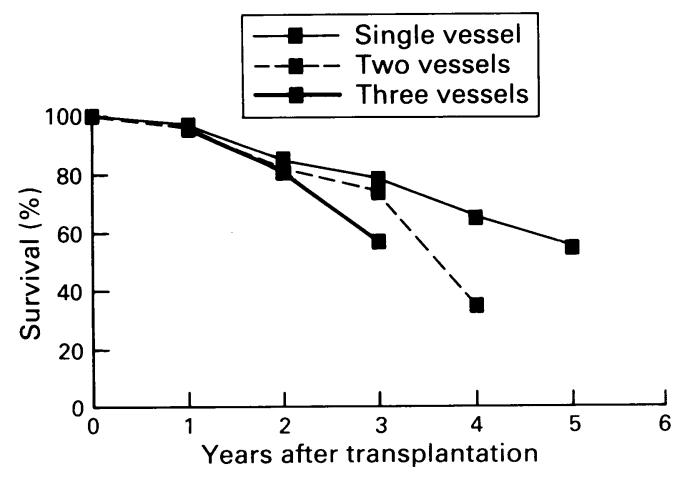

becomes clearer, appropriate preventive and therapeutic strategies are difficult to design.

Serial coronary angiography has been the main method of assessing coronary occlusive disease after cardiac transplantation. As a result of the previously described differences from conventional coronary artery disease, it underestimates the presence and severity of lesions. The reported prevalence of angiographic coronary occlusive disease after cardiac transplantation in other centres varies from between $2 \%$ and $18 \%$ at one year and $40 \%$ and $44 \%$ at five years. ${ }^{42324}$ The differences may partly represent variations in reporting criteria.

It is clear from our study that angiographic evidence of coronary disease does not always accurately predict the prognosis in an individual patient. The low number of graft failures from coronary occlusive disease in transplant patients limits the comparisons with the large trials undertaken in conventional coronary artery disease..$^{25}$ The apparently increased risk of death in cardiac transplant patients with coronary disease may be due to the disturbed relation between coronary arterial structure, as defined by angiography, with coronary vascular and myocardial function. The concentric luminal narrowing, sequential stenoses, the diffuse nature of the disease, and the involvement of small vessels are responsible for the functional impairment. The few patients who have died from coronary occlusive disease precludes identification of potential associated risk factors for death.

Coronary disease with acute coronary thrombosis in primary and secondary vessels caused death in seven of 13 available cases as shown at post mortem examination. Most of these atheromatous plaques (six of seven) had considerable severity of stenosis. Again, reservations must occur regarding the accuracy of measurements of stenosis with these post mortem findings. This experience of plaque rupture as a cause of sudden death, however, is similar to that seen in coronary atherosclerotic 
Table 3 Risk factors associated with coronary occlusive disease

\begin{tabular}{ll}
\hline Factor & Reference \\
\hline Hypertension & Becker et al ${ }^{17}$ \\
Increased donor and recipient age & Sharples et al $^{18}$ \\
Ischaemic heart disease as indication for transplant & Sharples et al ${ }^{14}$ \\
HLA mismatch and & Pennock et al ${ }^{18}$ \\
Anti-HLA antibodies & Reemtsma et al \\
Cytotoxic B cell antibodies & Hess et al $^{20}$ \\
Haemostatic factors (fibrinogen, VIIc, VIIIc) & Hunt et al $^{21}$ \\
\hline
\end{tabular}

disease in non-transplant patients. For example, in a series of 100 consecutive sudden coronary deaths, $44 \%$ had a $>50 \%$ coronary arterial stenosis with a thrombosis. ${ }^{26}$ It is also evident that most of the patients had considerable cardiomegaly. The cause of this is unclear, as the patients did not have untreated hypertension (two had treated hypertension). Presumably graft failure, at least in some patients, was a result of coronary occlusive disease in large and small vessels producing symptom free cardiac ischaemia in a denervated, hypertrophied heart. This resulted in myocardial infarction in some cases, and presumably, fatal arrythmias in all cases.

Coronary occlusive disease can produce several outcomes. Sudden plaque rupture is unpredictable and leads to infarction or death in both conventional and transplant coronary disease. ${ }^{5}$ In progressive coronary occlusive disease leading to left ventricular dysfunction, full assessment for further treatment can be made. At present, cardiac retransplantation is the only established treatment. Unfortunately, survival outcome is poorer than for first time transplant patients, ${ }^{1}$ and the recurrence rates of coronary occlusive disease are higher. ${ }^{27}$ Justifying such treatment in the face of acute donor shortage is dificult. ${ }^{28}$ Percutaneous transluminal coronary angioplasty can be used to dilate discrete lesions in the proximal coronary arteries in cardiac transplant patients. The short-term results are comparable with the effectiveness in conventional atherosclerosis. ${ }^{29}$ Its long-term impact on cardiac function and prognosis is unclear. The use of coronary artery bypass grafting has been limited, as distal coronary vessels suitable for surgery are rare. ${ }^{30}$

The lack of suitable treatment for coronary occlusive disease calls into question the clinical value of routine coronary angiography in the follow up of symptom free cardiac transplant patients after operation. This contrasts with its research role, where it is clear that although more patients are required, risk factor analysis may eventually provide insights into associated cofactors in development of disease. The presence of severe lesions in larger coronary arteries is likely to be associated with a poor prognosis as suggested by our study. The mortality data, however, suggests that moderate epicardial coronary disease and disease in smaller vessels are also important in the increased mortality in long-term heart transplant patients. Ventricular hypertrophy may also be an important risk factor.

Non-invasive methods of detection of coronary occlusive disease have been disappointing. ${ }^{31}$ We should therefore attempt to use newer methods of measuring impairment of myocardial perfusion and function produced by coronary occlusive disease. ${ }^{32}$ With this information, improved preventive and therapeutic strategies for long-term cardiac transplant patients with coronary occlusive disease could be made.

In conclusion coronary occlusive disease is the main cause of late mortality and morbidity in cardiac transplant patients. Coronary disease affecting primary and secondary coronary arteries is the main factor in late mortality after cardiac transplantation, although disease in smaller distal vessels and ventricular hypertrophy are also implicated. Improved methods of measuring impairment of myocardial perfusion and function produced by coronary occlusive disease should be developed.

1 Kriett JM, Kaye MP. The registry of the international heart transplant registry seventh official report. J Heart Lung Transplant 1990;9:323-36.

Billingham ME. Cardiac transplant atherosclerosis. Trans plant Proc 1987;4(suppl 5):19-25.

3 Johnson DE, Gao SZ, Schroeder JS, De Campli WM, Billingham ME. The spectrum of coronary artery pathologic findings in human cardiac allografts. $J$ Heart Transplant 1989;8(5):349-59.

4 Gao SZ, Alderman EL, Schroeder JS, Silverman JF, Hunt Gao SZ, Alderman EL, Schroeder J S, Silverman JF, Hunt SA. Accelerated coronary vascular disease in the heart transplant patient: coronary arter

5 Gao SZ, JS Schroeder, SA Hunt, ME Billingham, HA Gao SZ, JS Schroeder, SA Hunt, ME Billingham, HA
Valantine, EB Stinson. Acute myocardial infarction in cardiac transplant patients. Am J Cardiol 1989;64:1093-7.

6 Pomerance A, Davies MJ. In: The pathology of the heart. Oxford: Blackwell Scientific Publications, 1975:45.

7 Schroeder JS, Hunt SA. Chest pain in heart transplant patients. N Eng J Med 1991;324:1805-6.

8 Kountz SL, Williams MA, Williams PL, Kapros C, Dempster WJ. Mechanism of rejection of homotransplanted kidneys. Nature 1963;199:257-99.

9 Zusman DR, Stinson EB, Oyer PE, Baldwin JC, Jamieson SW, McGregor CG, et al. Determinants of accelerated graft atherosclerosis (AGAS) in conventional and cyclosporine treated heart transplant recipients [abstract]. sporine treated heart transplant Transplant 1985;4:587.

10 Narrod J, Kormos R, Armitage J, Hardesty R, Ladowski J, Griffith $B$. Acute rejection and coronary artery disease in Griffith B. Acute rejection and coronary artery disease in long-term survivors of heart

11 Hammond EH, Yowell RL, Nunoda S, et al. Vascular (humoral) rejection in heart transplantation: pathologic observations and clinical implications. J Heart Transplant 1989;8:430-43.

12 Ratkovec RM, Wray RB, Renlund DG, O'Connell JB, Bristow MR, Gay WA, et al. Influence of corticosteroidfree maintenance immunosuppression on allograft coronary artery disease after cardiac transplantation. $J$ Thorac Cardiovasc Surg 1990;100:6-12.

13 Stamler JS, Vaughan DE, Rudd MA, et al. Frequency of hypercholesterolaemia after cardiac transplantation. $\mathrm{Am} J$ Cardiol 1988;62:1268-72.

14 Sharples LD, Caine N, Mullins P, Scott JP, Solis E, English TAH, et al. Risk factor analysis for the major hazards following heart transplantation-rejection, infection and coronary occlusive disease. Transplantation 1991;52: coronary

15 Gao SZ, Schroeder IS, Alderman EL, Hunt SA, Valantine HA, Wiedrehold V, Stinson EB. Prevalence of accelerated HA, Wiedrehold V, Stinson EB. Prevalence of accelerated coronary artery disease in heart transplant survivors. Comparison of cyclosporine

16 Grattan MT, Moreno-Cabral CE, Starnes VA, Oyer PE, Stinson EB, Shumway NE. Cytomegalovirus infection is associated with cardiac allograft rejection and atherosclerosis. JAMA 1989;261:3561-6.

17 Becker DM, Markakis BS, Sension M, Vitali S, Baughman $\mathrm{K}$, Pearson TA. Hyperlipidaemia and hypertension following heart transplantation: potential causes of coronary atherosclerosis [abstract]. J Am Coll Cardiol 1986;7:9.

18 Pennock JL, Oyer PE, Reitz BA, Jamieson SW, Bieber CP, Wallwork $\mathrm{J}$, et al. Cardiac transplantation in perspective for the future: survival, complications, rehabilitation and cost. J Thorac Cardioavasc Surg 1982;83:168-77.

19 Reemtsma K. Vascular immunoobliterative disease: a common cause of graft failure. Transplant Proc 1989;21:3706.

20 Hess ML, Hastillo A, Mohanakumar T, Cowley MJ, Vetrovac G, Szentpery S, et al. Accelerated atherosclerosis in cardiac transplantation: role of cytotoxic B-cell antibodies and hyperlipidaemia. Circulation 1983;68:

21 Hunt B, Segal H, Yacoub M. Haemostatic changes after 
heart transplantation and their relationship to accelerated coronary atherosclerosis. Transplant Proc 1991;23: 1233-5.

22 Stovin PG, Sharples L, Hutter JA, Wallwork J, English TAH. Some prognostic factors for the development of transplant-related coronary artery disease in human cartransplant-related coronary artery disease in human car-

23 O'Neill BJ, Pflugfelder PW, Singh NR, Menkis AH, M'cKenzie FN, Kostuk WJ. Frequency of angiographic detection and quantitative assessment of coronary arterial disease one and three years after cardiac transplantation. Am J Cardiol 1989;63:1221-6.

24 Uretsky BF, Murali S, Reddy PS, Rabin B, Lee A, Griffith $\mathrm{BP}$, et al. Development of coronary artery disease in cardiac transplant patients receiving immunosuppressive therapy with cyclosporine and prednisone. Circulation 1987;76:827-34

25 Killip T, Passami E, Davis K, et al. Coronary artery surgery study: a randomized trial of coronary bypass surgery. Eight year follow-up and survival in patients with reduced ejection fraction. Circulation 1985;72(suppl V):102-12.

26 Davies MJ, Thomas A. Thrombosis and acute coronary lesions in sudden cardiac ischaemic death. $N$ Engl J Med 1984;310:1137.
27 Gao SZ, Schroeder JS, Hunt SA, Stinson EB. Retransplantation for severe accelerated coronary artery disease in heart transplant recipients. Am J Cardiol 1988;62:876-81.

28 Mullins PA, Scott JP, Dunning JJ, Aravot DJ, Large SR, Wallwork J, Schofield PM. Cardiac transplant waiting lists, donor shortage and retransplantation and implications for using donor hearts. Am J Cardiol 1991;68:

29 Copeland JG, Butman SM, Sethi G. Successful coronary artery bypass grafting for high-risk left main coronary artery stenosis after cardiac transplantation. Ann Thorac Surg 1991;49:106-10

30 Mullins PA, Shapiro LM, Aravot DJ, Scott JP, Large SR, Wallwork J, Schofield PM. Experience of PTCA in orthotopic cardiac transplant recipients. Eur Heart $J$ 1991;12:1205-8.

31 Smart FW, Ballantyne CM, Cocanougher B, Farmer JA, Sekela ME, Noon GP, Young JB. Insensitivity of noninvasive tests to detect coronary artery vasculopathy after heart transplant. Am J Cardiol 1991;67:243-7.

32 Mullins PA, Scott JP, Aravot DJ, Large SR, Wallwork J, Schofield PM. Small vessel coronary occlusive disease after cardiac transplantation [abstract]. J Am Coll Cardiol 1991;17:308. 